\title{
Local Approach to Fracture Based Prediction of Reactor Pressure Vessel Lifetime
}

\section{S. A. Kotrechko and Yu. Ya. Meshkov}

Kurdyumov Institute for Metal Physics, National Academy of Sciences of Ukraine, Kiev, Ukraine

New version of the local approach to fracture is presented. Within the framework of this approach a new methodology is developed, which supposes prediction of radiation life time of a reactor pressure vessel not by ultimate shift of the Charpy critical temperature, $\Delta T_{K}$, or by reference temperature, $\Delta T_{0}$, but by the condition of brittle fracture initiation of irradiated metal ahead of a crack tip in reactor pressure vessels.

Keywords: local approach, reactor pressure vessel (RPV) steels, RPV lifetime, fracture toughness, local stress.

Introduction. A critical-temperature-shift-based methodology is used for assessment of irradiation embrittlement of pressure vessel steels. Conventionally, the Charpy temperature shift methodology is utilized. Moreover, the attempts are made to use the Master curve technique [1] to solve this problem. Recently, the local approach to fracture (LAF) has been developed [2], which can be used as a powerful tool for solving this problem [3].

The aim of this study is to present a new version of the local approach to fracture and to exhibit ability of its application to predict pressure vessel lifetime.

Theory. In the general case, the statistical criterion of brittle fracture of a specimen or a structural element with a crack may be presented as

$$
F_{\Sigma}=1-\prod_{1}^{M}\left(1-F_{i}\right)
$$

where $F_{\Sigma}$ is the value of tolerance for the probability of fracture of the total cracked body, $F_{i}$ is the probability of fracture of an elementary volume, and $M$ is the number of such elementary volumes within the "process zone", i.e., within the region where the crack nuclei form.

The value of $F_{i}$ is usually assessed on the base of the weakest link principle, which gives the approximate equation:

$$
F_{i} \approx 1-\exp \left[-\rho_{i} V_{i}\left(\frac{\sigma_{1}^{i}-\sigma_{t h}}{\sigma_{u}}\right)^{m}\right] \text {, }
$$

where $\rho_{i}$ is the rate of the crack nuclei $(\mathrm{CN})$ generation within the volume unit of metal at given value of plastic strain, $V_{i}$ is the elementary volume, $\sigma_{1}^{i}$ is the 
maximum tensile stress within $i$ th elementary volume, and $\sigma_{t h}, \sigma_{u}$, and $m$ are the parameters of the Weibull distribution.

To ascertain relation between the value of local stress $\sigma_{1}^{i}$ at the moment of fracture and the macroscopic characteristic of loading, for instance, $K_{J_{c}}$, a nonlinear boundary value problem for a cracked body should be solved. The finite element method (FEM) enables one to find the value of $K_{J_{C}}$ with a priori given probability of fracture $F_{\Sigma}$. Such approach is realized in physical (ab initio) version of LAF presented in [3]. The difference is that probability of fracture of an elementary volume, $F_{i}$, was determined not by the approximate formula (2) but directly by computer simulation of the crack nuclei (CN) formation and instability.

However, analytical expression for the local fracture criterion is more acceptable for engineering calculations. As known for a cracked body, stress and strain distributions ahead of a crack tip are scaled in units $J_{\mathrm{I}} / \sigma_{Y}$, where $J_{\mathrm{I}}$ is the value of $J$-integral, and $\sigma_{Y}$ is the yield strength. It means that at the fixed value of $J_{\mathrm{I}} / \sigma_{Y}$, an unambiguous relation between the maximum value of the local probability of fracture initiation ahead of a crack, $F_{i}^{\max }$, and the total probability of fracture of a cracked body $F_{\Sigma}$, must exist. It enables one, using (2), to obtain the expression for the value of local strength of metal, $\sigma_{f}$, at given value of general probability $F_{\Sigma}$ :

$$
\sigma_{f}=\sigma_{t h}+\sigma_{u}\left[-\frac{\ln \left(1-F_{i}^{\max }\right)}{\rho_{i} V_{i}}\right]^{1 / m} .
$$

Respectively, the criterion of fracture initiation may be presented as

$$
\frac{\sigma_{f}}{\sigma_{1}} \leq 1
$$

In [4], it is shown that the value of the above ratio excess over the unit characterizes stability of ductile state of metal in the crack vicinity, i.e., its remoteness from brittle fracture. Thus, a new characteristic - a parameter of mechanical stability $P_{m s}$ - was proposed:

$$
P_{m s}=\frac{\sigma_{f}}{\sigma_{1}}
$$

At $P_{m s}>1$ the metal is relatively stable to brittle fracture, whereas at $P_{m s} \leq 1$ it is unstable (which means brittle fracture initiation).

As shown in [4], the value of the metal local strength, $\sigma_{f}$, can be expressed via $R_{m c}$ - the minimum value of fracture stress of a standard specimen under uniaxial tension within the ductile-to-brittle-transition temperature range:

$$
\sigma_{f}=k_{v} R_{m c}
$$


where coefficient $k_{v}$ is the measure of the scale effect related to excess of the value of fracture stress $\sigma_{f}$ of the local volume $V_{i}$ over the minimum stress of brittle fracture of standard tensile specimen $\left(V_{0} \approx 1000 \mathrm{~mm}^{3}\right), R_{m c}$. According to (3), the expression for $k_{v}$ is

$$
k_{v}=\frac{\sigma_{t h}}{R_{m c}}+\frac{\sigma_{u}}{R_{m c}}\left[-\frac{\ln \left(1-F_{i}^{\max }\right)}{\rho_{i} V_{i}}\right]^{1 / m} .
$$
follows:

The tensile stress level ahead of the crack tip, $\sigma_{1}$, may be presented as

$$
\sigma_{1}=j \sigma_{Y}\left(\frac{e_{f}}{e_{Y}}\right)^{n}
$$

where $j$ is the coefficient of local overstress $\left(j=\sigma_{1} / \bar{\sigma}\right.$, where $\bar{\sigma}$ is equivalent stress), $e_{f}$ is the value of local plastic strain within the local region where $F_{i}=F_{i}^{\max }, e_{Y}$ is plastic strain at yield strength, and $n$ is strain-hardening exponent.

Accounting for (6) and (8), the expression for $P_{m s}$ is

$$
P_{m s}=\frac{K_{m s}}{q_{c r}},
$$

where $K_{m s}$ is the coefficient of mechanical stability of metal under uniaxial tension:

$$
K_{m s}=\frac{R_{m c}}{\sigma_{Y}\left(e_{c} / e_{Y}\right)^{n}}
$$

$q_{c r}$ is the force equivalent of the embrittlement effect due to both triaxial tension and localization of fracture initiation ahead of the crack tip,

$$
q_{c r}=\frac{j}{k_{v}}\left(\frac{e_{f}}{e_{c}}\right)^{n},
$$

$K_{m s}$ characterizes the level of stability of the metal plastic state under uniaxial tension, and is unambiguously determined by the mechanical properties obtained under uniaxial tension: $R_{m c}, \sigma_{Y}$, and $n$. Parameter $q_{c r}$ shows how will this initial level of stability decrease if this metal is placed at the macrocrack tip [4] For typical pressure vessel steels $\sigma_{Y}=\sigma_{0.2}\left(e_{Y}=0.002\right)$, and critical strain is $e_{c} \approx 0.02$, therefore, $K_{m s}=R_{m c} /\left(\sigma_{0.2} \cdot 10^{n}\right)$.

According to (10), radiation-enhanced hardening of pressure vessel steel must result in the reduction of it mechanical stability level, $K_{m s}$. Using Eqs. (9) and (10) and well-known dependence yield strength increment $\Delta \sigma_{Y s}$ vs. fluence 
value $\Phi\left(\Delta \sigma \approx B_{h}\left(\Phi / 10^{22}\right)^{m_{\Phi}}\right.$, where $m_{\Phi} \approx 0.33-0.51$ [5]), and the expression for the parameter of mechanical stability of irradiated steel, $P_{m s}^{i r}$, can be obtained

$$
P_{m s}^{i r}=\frac{K_{m s}}{E_{m}}
$$

where $E_{m}$ is the general force equivalent of the embrittlement which characterizes the total embrittlement due to both stress-strain field ahead of a crack tip and radiation-enhanced hardening of metal:

$$
E_{m s}=q_{c r} q_{i r},
$$

where coefficient $q_{\text {ir }}$ characterizes the degree of radiation embrittlement of steel due to it radiation-enhanced hardening [4]:

$$
q_{i r} \approx 1+\frac{B_{h}}{\sigma_{Y}}\left(\frac{\Phi}{10^{22}}\right)^{m_{\Phi}}
$$

Dependences (12) and (14) enable one to predict the value of critical fluence $\Phi_{c}$, at which the cracked pressure vessel fails $\left(P_{m s}^{i r}=1\right)$ at the given value of load $J_{\mathrm{I}} / \sigma_{Y}$.

When solving this problem, the scale effect is critical. According to (11) and (7), this effect governs the embrittling force value of a crack (parameter $q_{c r}$ ).

In the first approximation, the value of an elementary volume $V_{i}$ can be expressed as

$$
V_{i}=B h_{i}
$$

where $B$ is the crack front length and $h_{i}$ is parameter characterizing the width of an elementary volume, for which $\sigma_{f}$ is determined. The upper limit of $h_{i}$ is determined from the condition that stress and strain variations within $h_{i}$ limits may be neglected. The lower limit of $h_{i}$ characterizes the minimum size of the region required for the $\mathrm{CN}$ formation. In the first approximation, this size must be of the true grain size order.

Accounting for (15) and (7), the expression (11) for $q_{c r}$ will be the following:

$$
q_{c r}=\frac{j\left(e_{f} / e_{c}\right)^{n}}{\left\{\frac{\sigma_{t h}}{R_{m c}}+\frac{\sigma_{u}}{R_{m c}}\left[-\frac{\ln \left(1-F_{i}^{\max }\right)}{\rho_{i} h_{i} B}\right]^{1 / m}\right\}}
$$

According to (16), susceptibility of the value of embrittling force of a crack $q_{c r}$ to change in the crack front length $B$, depends on the value of parameter $m$. For typical pressure vessel steels, $m$ is determined, above all, by the inhomogeneity of distribution of carbide sizes. 
Technique for Prediction of Critical Fluence $\Phi_{c}$. The essence of technique proposed is to obtain the dependence of the parameter of mechanical stability $P_{m s}$ on the fluence value $\Phi$, and to find critical value $\Phi_{c}$, at which $P_{m s}=1$ (i.e., brittle fracture is initiated ahead of a crack-like defect tip in a pressure vessel) grounded on the findings of tests of surveillance specimens. According to (12), it is necessary to know the value of $K_{m s}$ for steel in the initial (non-irradiated) state and then to estimate the degree of irradiation embrittlement of steel $\left(q_{i r}\right)$ grounded on the findings of tests of surveillance specimens under uniaxial tension as well as to find the value of $q_{c r}$ for a cracked non-irradiated metal.

As it follows from (10), brittle strength of metal, $R_{m c}$, determined as the minimum fracture stress over the ductile-to-brittle-transition temperature region under uniaxial tension [4] is the key characteristic for $K_{m s}$ determination.

Figure 1 presents the dependences of $K_{m s}^{i r}$ and $q_{i r}$ on fluence for pressure vessel steel 15Kh2NMFA (Table 1).

$\mathrm{T}$ a $\mathrm{b} 1 \mathrm{e} 1$

Mechanical Properties of Pressure Vessel Steel 15Kh2NMFA in the Initial and Irradiated States

\begin{tabular}{|c|c|c|c|c|c|c|c|}
\hline \multirow{2}{*}{ Steel } & \multirow{2}{*}{$\begin{array}{c}R_{m c} \\
\mathrm{MPa}\end{array}$} & \multicolumn{3}{|c|}{ Non-irradiated state } & \multicolumn{4}{|c|}{ Irradiated state } \\
\cline { 3 - 9 } & $\begin{array}{c}\sigma_{0.2,2}, \mathrm{MPa} \\
\left(20^{\circ} \mathrm{C}\right)\end{array}$ & $\begin{array}{c}n \\
\left(20^{\circ} \mathrm{C}\right)\end{array}$ & $T_{0},{ }^{\circ} \mathrm{C}$ & $\begin{array}{c}\sigma_{02}^{i r}, \mathrm{MPa} \\
\left(20^{\circ} \mathrm{C}\right)\end{array}$ & $\begin{array}{c}n \\
\left(20^{\circ} \mathrm{C}\right)\end{array}$ & $T_{0},{ }^{\circ} \mathrm{C}$ \\
\hline 15Kh2NMFA & 1400 & 595 & 0.06 & -167 & 663 & 0.05 & -130 \\
\hline
\end{tabular}

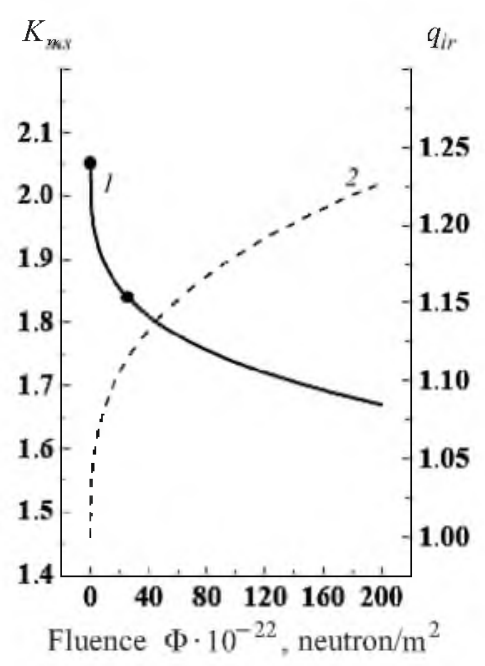

Fig. 1. Effect of the fluence value $\Phi$ on the coefficient of mechanical stability $K_{m s}(I)$ and the parameter $q_{i r}$ (2) of RPV steel 15Kh2NMFA.

The essence of technique of experimental determination of $q_{c r}$ value for a crack in non-irradiated steel is shown in Fig. 2. It consists in the ascertainment of critical temperature $T_{c}$, at which fracture of specimen occurs at specified value of $J_{\mathrm{I}} / \sigma_{Y}$. The point of intersection of the temperature dependences of fracture 
toughness of non-irradiated steel, $K_{J c}$, and stress intensity coefficient $K_{I L}$ for the given value $J_{\mathrm{I}} / \sigma_{Y}$ determines the above temperature. In this case:

$$
K_{L}=\sqrt{\frac{E \sigma_{Y}}{1-v^{2}} b L}
$$

where $L$ is the dimensionless loading parameter:

$$
L=1 / M=J_{\mathrm{I}} /\left(b \sigma_{Y}\right)
$$

$M$ is the dimensionless parameter in ASTM E1921, $b$ is the ligament size, $E$ is the Young modulus, and $v$ is Poisson's ratio.

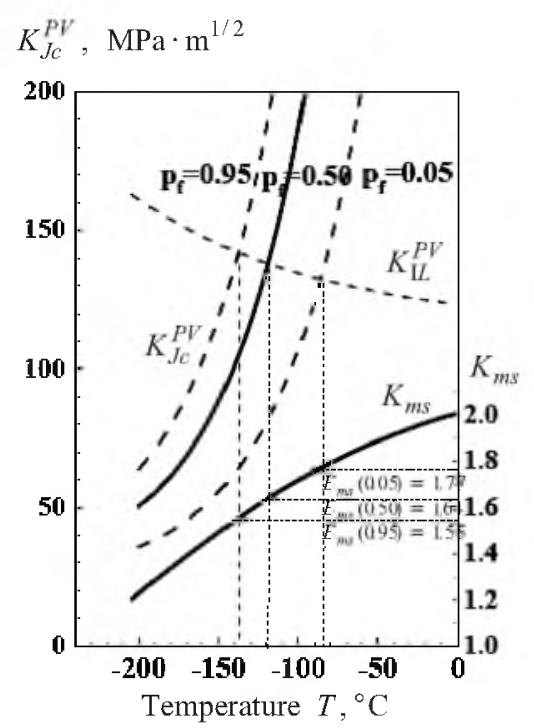

Fig. 2. The temperature dependence of the coefficient of mechanical stability of pressure vessel steel, $K_{m s}$, and the value $K_{J c}^{P V}$ for proof crack in a reactor pressure vessel.

The value of parameter $q_{c r}$ is quantitatively equal to the magnitude of $K_{m s}$ at this critical temperature $T_{c}$. It follows from the dependence (9), according to which condition $q_{c r}=K_{m s}\left(T_{c}\right)$ holds at the moment of fracture $\left(P_{m s}=1\right)$. The values of $q_{c r}$ for fracture probabilities 0.05 and 0.95 are ascertained similarly.

Knowing the coefficient of mechanical stability in the initial state, $K_{m s}$, the value of parameter, $q_{c r}$, and the relation between $q_{i r}$ and $\Phi$, the dependence of parameter of mechanical stability of irradiated steel, $P_{m s}^{i r}$, on the fluence value can be plotted (Fig. 3). The value of fluence, at which $P_{m s}^{i r}=1$, is the critical value of $\Phi=\Phi_{c}$.

Results and Discussion. Pressure vessel steel 15Kh2NMFA in the irradiated and initial states was employed as the subject of study (Table 1).* Figure 1 presents dependences of the coefficient of mechanical stability $K_{m s}^{i r}$ and $q_{i r}$ on

* Mechanical tests were conducted by Senior Researcher, Ph.D. V. N. Revka. 
the value of fluence at the temperature $20^{\circ} \mathrm{C}$. To approximate the experimental data, the value $m_{\Phi}=1 / 3$ was used. Figure 2 demonstrates the technique of determination of the parameter $q_{c r}$. Temperature dependence of $K_{J c}$ was plotted based on the test data on three-point bending of precracked surveillance Charpy specimens. The value of $K_{J c}$ was adjusted to $B=150 \mathrm{~mm}$ by the Master curve technique (ASTM E1921 standard). This $B$ value is approximately equal to the front length of a proof elliptic crack of depth $a=25 \mathrm{~mm}(0.125 S$, where $S$ is a thickness of the reactor pressure vessel wall). To simplify calculations, curvilinearity of the crack front was neglected. Calculations were executed for $J_{\mathrm{I}} / \sigma_{Y}=0.13 \mathrm{~mm}$.

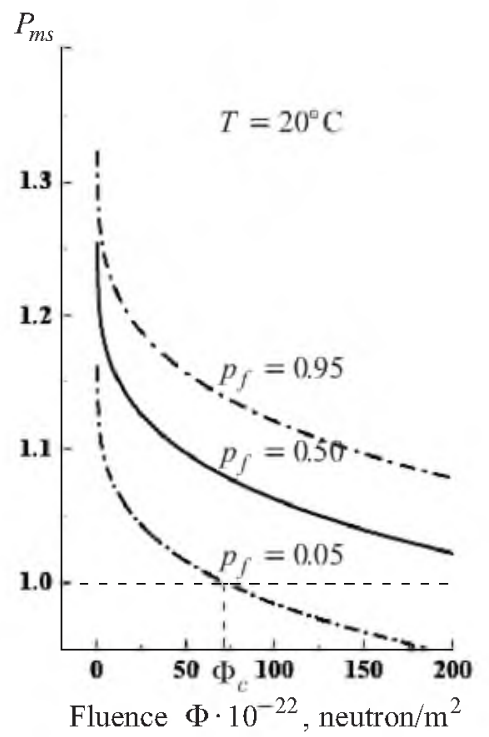

Fig. 3. Effect of the fluence value, $\Phi$, on the parameter of mechanical stability of steel, $P_{m s}$, ahead of a crack tip in a reactor pressure vessel wall.

According to calculation results, at this loading level $\left(J_{\mathrm{I}} / \sigma_{Y}=0.13 \mathrm{~mm}\right)$ the effect of the crack embrittlement parameter for given values of probabilities $0.05 ; 0.50$, and 0.95 amounts to $q_{c r}^{0.05}=1.765, q_{c r}^{0.50}=1.635$, and $q_{c r}^{0.95}=1.550$, respectively. Data presented in Figs. 1 and 2 enable one to plot the dependence of the mechanical stability parameter $P_{m s}$ on the fluence value and to ascertain the critical magnitude of the fluence (Fig. 3). Calculation results clearly manifest the essential effect of the probability of critical event under consideration on the critical fluence value. Thus, at the probability of unstable equilibrium of a crack-like defect in a pressure vessel $p_{f}=0.5$, the value of critical fluence exceeds $\Phi_{c}>200 \cdot 10^{22}$ neutron $/ \mathrm{m}^{2}$, and at $p_{f}=0.05$, the critical fluence value amounts to $\Phi_{c}=72 \cdot 10^{22}$ neutron $/ \mathrm{m}^{2}$, which is much closer to the standard value of 57 neutron $/ \mathrm{m}^{2}$ for pressure vessels of WWER-1000 reactors. Noteworthy is that option of determination of the critical fluence value with a priori probability makes possible a quantitative prediction of the reliability of safe operation of a reactor pressure vessel. 


\section{Conclusions}

1. A new approach to prediction of reactor pressure vessel lifetime is proposed; within the framework of this approach, the critical value of fluence is determined not by the ultimate shift of Charpy critical temperature, $\Delta T_{K}$, or by $\Delta T_{0}$ according to the Master curve technique, but by the condition of instability of the ductile state (initiation of brittle fracture) of irradiated metal ahead of a crack tip in a reactor pressure vessel.

2. The condition of ductility exhaustion (initiation of brittle fracture) of the irradiated metal within the local region ahead of a crack tip can be described by two new mechanical characteristics, namely:

(i) the coefficient of mechanical stability $K_{m s}$, which characterizes ability of metal to resist transition from ductile to brittle state in laboratory conditions of uniaxial tension; it is determined unambiguously by such structure-sensitive characteristics as brittle strength $R_{m c}$, yield strength $\sigma_{0.2}$, and strain-hardening exponent $n\left(K_{m s}=R_{m c} /\left(\sigma_{0.2} \cdot 10^{n}\right)\right)$;

(ii) force equivalent of embrittlement $E_{m}$, which demonstrates how much is this initial (laboratory) level of mechanical stability, $K_{m s}$, decreased by the effect of both radiation strengthening of steel (parameter $q_{i r}$ ) and inhomogeneous force field ahead of a crack tip (parameter $\left.q_{c r}\right)\left(E_{m}=q_{i r} q_{c r}\right)$.

1. W. Server, S. Rosinski, R. Lott, et al., "Application of Master Curve fracture toughness for reactor pressure vessel integrity assessment in the USA," Int. J. Press. Vess. Piping, 79, 701-713 (2002).

2. A. Pineau, "Development of the local approach to fracture over the past 25 years: theory and applications," Int. J. Fract., 138, 139-166 (2006).

3. S. Kotrechko, "Physical fundamentals of local approach to analysis of cleavage fracture," in: Transferability of Fracture Mechanical Characteristics, Kluwer Academic Publishers (2002), pp.135-150.

4. S. Kotrechko and Yu. Meshkov, "A new approach to estimate irradiation embrittlement of pressure vessel steels," Int. J. Press. Vess. Piping, 85, 336-343 (2008).

5. T. Byur and K. Farell, "Irradiation hardening behavior of polycrystalline metals after low temperature irradiation," J. Nucl. Mater., 326, 86-96 (2004). 